\title{
Severe Oleander Poisoning Presenting with Hyperkalaemia and Unusual Electrocardiographic Changes
}

\author{
Satyabrata Guru, Anupama Behera, Sadananda Barik, Upendra Hansdah, Chitta R Mohanty, Sangeeta Sahoo \\ All India Insititute of Medica Sciences, Bhubaneswar, Odisha, India
}

Doi: 10.12890/2021_003044- European Journal of Case Reports in Internal Medicine - ๔ EFIM 2021

Received: 02/11/2021

Accepted: 22/11/2021

Published: $14 / 12 / 2021$

How to cite this article: Guru S, Behera A, Barik S, Hansdah U, Mohanty CR, Sahoo S. Severe oleander poisoning presenting with hyperkalaemia and unusual electrocardiographic changes. EJCRIM 2021;8: doi:10.12890/2021_003044.

Conflicts of Interests: The authors declare there are no competing interests.

This article is licensed under a Commons Attribution Non-Commercial 4.0 License

\section{ABSTRACT}

Background:Hyperkalaemiainoleander(Neriumoleander)poisoninghas beenassociated withapoor prognosis. Differentelectrocardiographic (ECG) presentations are possible because of vagotonia and hyperkalaemia.

Methods/Results: We report a series of three cases of oleander poisoning in which ECG showed unusual hyperkalaemia features, such as bradyarrhythmia, sinoatrial block, atrioventricular block and junctional rhythm.

Conclusions: If arterial blood gas analysis or laboratory values indicate hyperkalaemia in oleander poisoning, the hyperkalaemia should be treated immediately, even if the ECG does not show typical hyperkalaemia features.

\section{LEARNING POINTS}

- Hyperkalaemia in oleander poisoning is associated with a poor prognosis.

- Hyperkalaemia should be treated aggressively in oleander poisoning even if the ECG does not show typical features, as mimics other conditions on ECG.

\section{KEYWORDS}

ECG, hyperkalaemia, oleander poisoning

\section{INTRODUCTION}

Oleander (Nerium oleander) is an extremely poisonous plant with a non-digitalis cardiac glycoside effect. All parts of the plant are poisonous including the leaves, roots and seeds. Most symptoms from oleander poisoning are cardiac and gastrointestinal in nature and appear 4 hours after ingestion ${ }^{[1]}$. The $\mathrm{Na}+/ \mathrm{K}+$ ATPase pump of the heart is affected, resulting in hyperkalaemia and a direct cardiac glycoside poisoning effect, causing vagotonia. The vagotonia and hyperkalaemia can cause different clinical features and electrocardiographic (ECG) presentations. We report three cases of oleander poisoning in which the typical ECG features of hyperkalaemia were absent but less common hyperkalaemia features were present (Table 1).

\section{CASE DESCRIPTIONS}

\section{Case 1}

A 44-year-old male patient presented to the emergency department (ED) 12 hours after ingesting four oleander seeds. His chief complaint was dizziness and a tingling sensation all over his body. On examination, he was conscious, pulse was 54/min, irregular, blood pressure (BP) 


\begin{tabular}{|c|c|c|c|}
\hline Case & Vital signs & Laboratory values & ECG features \\
\hline Case 1 & $\begin{array}{l}\text { HR 54/min } \\
\text { BP 120/54 mmHg } \\
\text { SpO2 98\% }\end{array}$ & $\begin{array}{l}\mathrm{Na}+135 \mathrm{mmol} / \mathrm{l} \\
\mathrm{K}+10.36 \mathrm{mmol} / \mathrm{l} \\
\mathrm{Ca} 2+2.1 \mathrm{mmol} / \mathrm{l} \\
\mathrm{Mg} 2+1.8 \mathrm{mg} / \mathrm{dl}\end{array}$ & $\begin{array}{l}\text { Sinus bradycardia with variable } \\
\text { AV block with short QT interval }\end{array}$ \\
\hline Case 2 & $\begin{array}{l}\text { HR 70/min } \\
\text { BP 100/60 mmHg } \\
\text { SpO2 100\% }\end{array}$ & $\begin{array}{l}\mathrm{Na}+146.8 \mathrm{mmol} / \mathrm{l} \\
\mathrm{K}+8.23 \mathrm{mmol} / \mathrm{l} \\
\mathrm{Ca} 2+2.3 \mathrm{mmol} / \mathrm{l} \\
\mathrm{Mg} 2+1.7 \mathrm{mg} / \mathrm{dl}\end{array}$ & $\begin{array}{l}\text { Sinus node dysfunction with } \\
\text { junctional escape rhythm and } \\
\text { ectopic atrial rhythm with short } \\
\text { QT interval }\end{array}$ \\
\hline Case 3 & $\begin{array}{l}\text { HR 95/min } \\
\text { BP 120/76 mmHg } \\
\text { SpO2 100\% }\end{array}$ & $\begin{array}{l}\mathrm{Na}+126.4 \mathrm{mmol} / \mathrm{l} \\
\mathrm{K}+7.24 \mathrm{mmol} / \mathrm{l} \\
\mathrm{Ca} 2+2.2 \mathrm{mmol} / \mathrm{l} \\
\mathrm{Mg} 2+2 \mathrm{mg} / \mathrm{dl}\end{array}$ & $\begin{array}{l}\text { P waves were small, T wave inver- } \\
\text { sion in leads II, III, aVF and V1-3, } \\
\text { QRS duration of } 77 \mathrm{~ms} \text { (narrow } \\
\text { QRS) }\end{array}$ \\
\hline
\end{tabular}

Table 1. Vital signs, laboratory data and electrocardiographic features of patients in this study

was $120 / 54 \mathrm{mmHg}$, and oxygen saturation (SpO2) was $98 \%$ on room air. Other systems were normal. Arterial blood gas (ABG) analysis showed K+ $10.36 \mathrm{mmol} / \mathrm{l}, \mathrm{Na}+135 \mathrm{mmol} / \mathrm{I}$ and Ca2 $1.1 \mathrm{mmol} / \mathrm{I}$. An ECG was immediately performed (Fig. 1) and revealed a heart rate (HR) of $53 / \mathrm{min}$, variable atrioventricular block (prolonged PR interval with variable atrioventricular block), and a corrected QT interval of 228 ms. As the ECG did not show the typical features of hyperkalaemia, we immediately repeated the ABG, which confirmed the hyperkalaemia. The patient received treatment for hyperkalaemia with $25 \%$ dextrose along with regular insulin, nebulization with salbutamol, and intravenous fluids. However, he experienced a sudden cardiac arrest, could not be revived, and died within 2 hours of presentation.

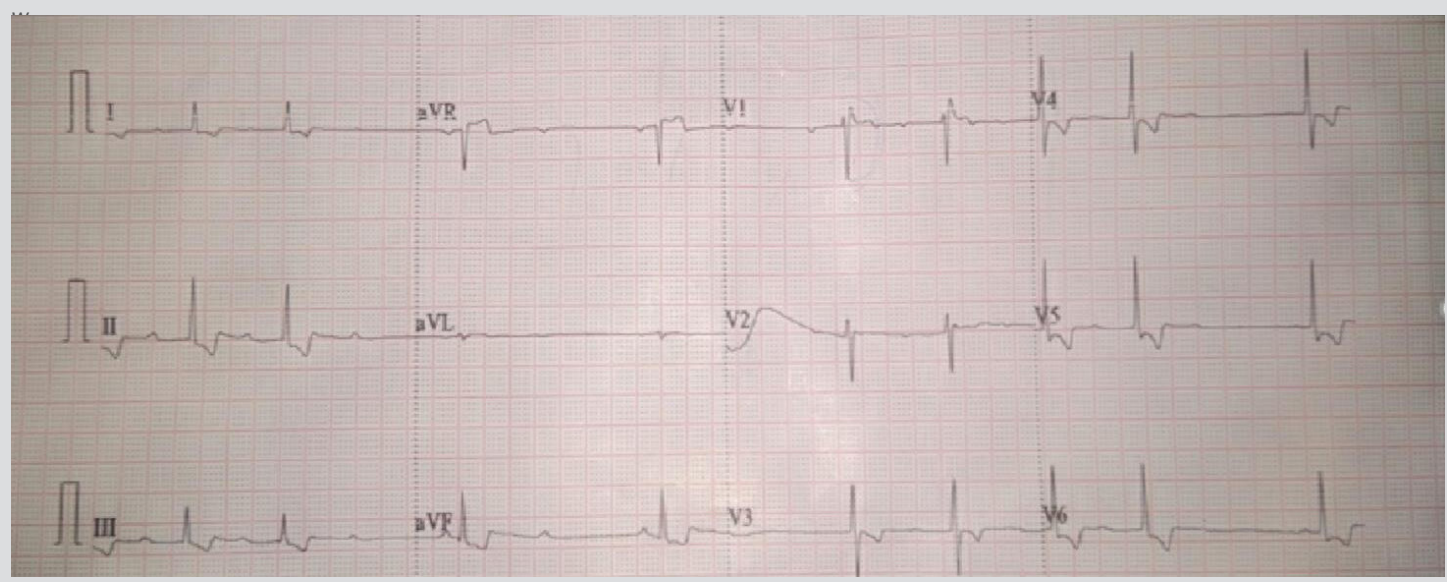

Figure 1. ECG for patient 1

\section{Case 2}

A 35-year male patient presented to the ED after ingesting three oleander seeds with a chief complaint of two episodes of vomiting. He was conscious, his pulse was 70/min, regular, BP was $100 / 60 \mathrm{mmHg}$, and SpO2 was $100 \%$ on room air. ABG showed K+ $8.23 \mathrm{mmol} / \mathrm{l}$, Na+ 146.8 $\mathrm{mmol} / \mathrm{l}$, and hyperkalaemia was confirmed with a repeat ABG. An ECG was immediately performed (Fig. 2) and revealed HR 60/min, with features of a digoxin effect such as the hockey stick sign, absent sinus $\mathrm{P}$ waves with some beats showing negative $\mathrm{P}$ waves preceding the QRS complexes and some showing absent P waves preceding the QRS complexes mainly in the inferior leads, and a short QT interval with a corrected QTc of 294-336 ms (i.e., sinus node dysfunction with junctional escape rhythm and ectopic atrial rhythm with a short QT interval), but the ECG did not show any of the common features of hyperkalaemia. The patient was treated for hyperkalaemia with $25 \%$ dextrose and regular insulin, salbutamol nebulization, and intravenous fluids. Hyperkalaemia was gradually corrected over 24 hours, and the patient was discharged asymptomatic after 72 hours. 


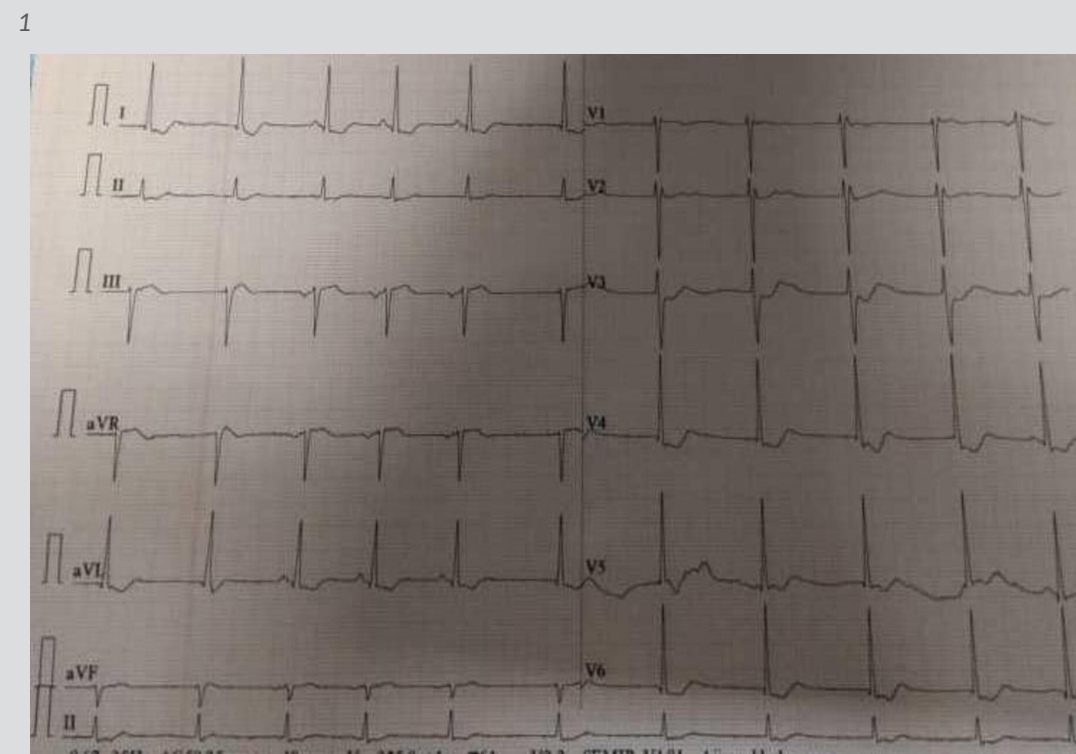

Figure 2. ECG for patient 2

Case 3

A 28-year woman presented to the ED having ingested one oleander seed. Her chief complaint was three episodes of vomiting. She was conscious, pulse rate was 95/minute, BP was 120/76 mm abnormalities. ABG showed K+ $7.24 \mathrm{mmol} / \mathrm{I}$ and $\mathrm{Na}+126.4 \mathrm{mmol} / \mathrm{I}$. An ECG was immediately performed (Fig. 3) to confirm hyperkalaemia. It showed showed HR 94/min, small P waves, T wave inversion in leads II, III, aVF and V1-3, and a QRS duration of 77 ms (narrow QRS). As the ECG showed less common hyperkalaemia features, a repeat ABG was done, and hyperkalaemia was confirmed. The patient was treated with pantoprazole, ondansetron and intravenous fluids. Hyperkalaemia was managed with $25 \%$ dextrose, regular insulin and salbutamol nebulization. Hyperkalaemia corrected over 12 hours and the patient was discharged after 72 hours.

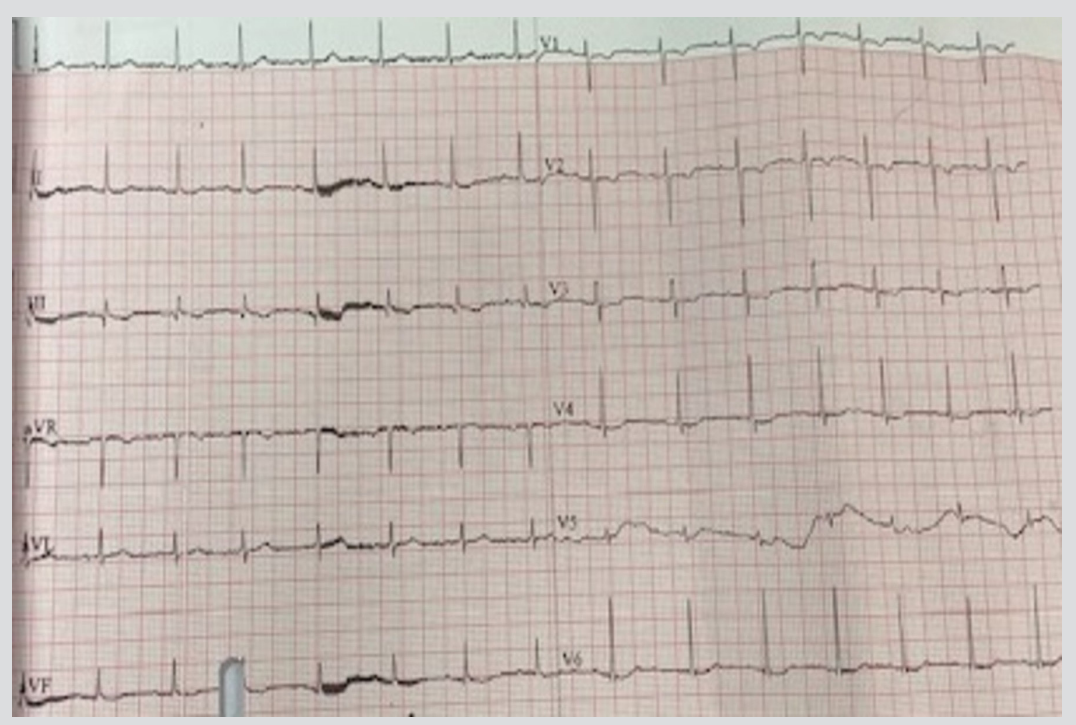

Figure 3. ECG for patient3

\section{DISCUSSION}

Oleander is used as an ornamental plant in India and Sri Lanka. All parts of the plant are poisonous, including the seeds and leaves. Ingestion is usually deliberate in order to commit suicide with some rare accidental cases in children. All three of our patients intended to commit suicide. Oleander contains oleandrin and nerine as toxic cardiac glycosides. 
The most serious side effects of oleander poisoning are cardiac abnormalities, such as ventricular dysrhythmias, bradycardia and heart block [2]. Hyperkalaemia in oleander poisoning is due to potassium shift to the extracellular space rather than an increase in total body potassium. The severity of poisoning mainly depends on cardiac involvement, in the form of heart block and arrhythmias and concomitant hyperkalaemia, which is an independent predictor of severe oleander poisoning ${ }^{[3]}$. All our patients had uncommon ECG features of hyperkalaemia and not typical ECG features, proving hyperkalaemia is a great mimicker on ECG. Although earlier case reports of oleander poisoning demonstrated markedly elevated potassium levels without significant ECG changes ${ }^{[4,5]}$, no previous reports in the literature have shown that oleander poisoning can present with an array of different and uncommon hyperkalaemia features on ECG.

Our first patient could not be revived and died, possibly because of refractory hyperkalaemia. Haemodialysis was not helpful in case of refractory hyperkalaemia due to oleander poisoning. In this case, digoxin-specific antibodies would have been lifesaving but were not available. The second and third cases had hyperkalaemia but recovered following conventional hyperkalaemia treatment. Aggressive treatment of hyperkalaemia in case of oleander poisoning is key. Treatment includes insulin-dextrose infusion, atropine, antiarrhythmic drugs, a temporary venous pacemaker, and administration of digoxin-specific Fab antibody fragments (Digibind) ${ }^{[6]}$. Intravenous calcium is not given due to the risk of arrhythmia. The inconsistent ECG manifestations may be due to cardiac myocyte adaptation tohyperkalaemia. The literature has shown that physicians correctly interpret ECG findings as indicating hyperkalaemia in only $50 \%$ of cases ${ }^{[7]}$. Hyperkalaemia in oleander poisoning is associated with a poor prognosis, and ECG to investigate hyperkalaemia may only show minimal or uncommon changes. Hence serum $\mathrm{K}+$ /ABG potassium should be assessed in case of oleander poisoning even if the ECG does not show typical hyperkalaemia features as oleander poisoning may mimic other conditions. In addition, lack of ECG changes consistent with typical hyperkalaemia should not preclude treatment. Detailed study of more cases is required in the future.

\section{CONCLUSION}

Hyperkalaemia in oleander poisoning should be treated aggressively even if the ECG does not show typical features because hyperkalaemia in oleander poisoning can mimic other conditions.

\section{REFERENCES}

Behcet Al, Yarbil P, Dogan M, Kabul S, Yildirm C. A case of non-fatal oleander poisoning. BMJ Case Rep 2010;2010:bcr02.2009.1573.

Ansford AJ, Morris H. Fatal oleander poisoning. Med J Aust 1981;1:360-1.

Alexandre J, Foucalt A, Coutance G, Scanu P, Milliez P. Digitalis intoxication induced by an acute accidental poisoning by lily of the valley. Circulation 2012;125(8):1053-1055. doi: 10.1161/CIRCULATIONAHA.111.044628.

4. Aslam S, Friedman EA, Ifudu O. Electrocardiography is unreliable in detecting potentially lethal hyperkalemia in hemodialysis patients. Nephrol Dial Transplant 2002;17:16391642.

5. Martinez-Vea A, Bardaji A, Garcia C, Oliver JA. Severe hyperkalemia with minimal electrocardiographic manifestations. A report of seven cases. J Electrocardiol 1999;32:4549.

6. Shumaik GM, Wu AW, Ping AC. Oleander poisoning: treatment with digoxin-specific Fab antibody fragments. Ann Emerg Med 1988;17:732-735.

7. Wrenn KD, Slovis CM, Slovis BS. The ability of physicians to predict hyperkalemia from the ECG. Ann Emerg Med 1991;20:1229-1232. 